\title{
A Comprehensive Map for Integrating Augmented Reality During the Construction Phase
}

\author{
$\underline{\text { Hala Nassereddine }}{ }^{1}, \underline{\text { Christian Schranz }}{ }^{2}$, Makram Bou Hatoum ${ }^{3}$ and Harald Urban ${ }^{4}$
}

1 University of Kentucky, Lexington, USA, hala.nassereddine@uky.edu

2 Technical University of Vienna, Austria, christian.schranz@tuwien.ac.at

3 University of Kentucky, Lexington, USA, mbh.93@uky.edu

4 Technical University of Vienna, Austria, harald.urban@tuwien.ac.at

\begin{abstract}
The construction industry has undergone a significant and radical transformation in its design and documentation process as it evolved from the days of the drafting board to today's Building Information Modeling (BIM) process. At each stop along that journey, gains were made in information density and exchange. However, for all the progress made thus far, the paradox of designing the 3D in 2D space remains. This paradox indicates that new visualization technologies are needed to leverage the use of information in construction. Augmented Reality (AR), a pillar of the fourth industrial revolution, is an emerging technology that has great potential to transform the construction industry. AR can be described as an information aggregator that allows the user to passively view displayed information, actively engage and interact with published content, and collaborate with others in real-time from remote locations. While AR holds the key to advance the construction industry, no research project has yet comprehensively investigated the holistic integration of AR in construction. The contribution of this paper to the body of knowledge is a comprehensive map that establishes a sound framework for specifying the appropriate integration of AR into the construction phase. The comprehensive map is based on the Task-Technology Fit theory, where 23 use-cases of AR in the construction phase are identified and outlined as a function of nine AR capabilities and 14 AR potential benefits. The AR use-cases, capabilities and potential benefits are first discussed in the paper. Then, two AR applications are explored where the underlying use-cases are discussed and mapped as a function of their corresponding AR capabilities and potential Benefits. These AR applications provide an example illustrating the concept behind the comprehensive map. Finally, the comprehensive map is developed to provide a holistic framework to understand the integration of AR into the construction phase.
\end{abstract}

(c) 2020 The Authors. Published by Budapest University of Technology and Economics \& Diamond Congress Ltd Peer-review under responsibility of the Scientific Committee of the Creative Construction Conference 2020.

Keywords: augmented reality, framework, taxonomy, task-technology fit, visualization

\section{Introduction and background}

Construction plays an important role in the prosperity of nations and is expected to grow to a global expenditure of $\$ 15.5$ trillion in 2030 [1]. This significant expansion along with the increased complexity and sophistication of construction projects and rapid advances in emerging technologies has fuelled companies' interest in innovation as a source of competitive advantage. Researchers argue that technology-enabled innovations can provide companies significant opportunities to maintain their vitality and competitive edge. One technology that has gained great interest in recent years is Augmented Reality (AR), one of the nine pillars of Industry 4.0. AR can be described both as an information aggregator and a data publishing platform that allows the user to (1) passively view displayed information, (2) actively engage and interact with published content, and (3) collaborate with others in real time from remote locations [2]. As industrial 
interest in AR has increased in the past decade (in sectors such as gaming, automotive, aerospace, military, and marketing), the construction industry has begun to follow suit in this area. AR is said to transform the construction industry and provide companies with a new frontier for gaining competitive advantage.

While previous research studies have investigated opportunities to integrated AR into the construction phase of a construction project, no research project has yet comprehensively assessed the relationship between the technology itself, the use-cases to be implemented, and the anticipated benefits. This study contributes to the body of knowledge by linking the AR capabilities to construction use-cases and connecting each use-case to the perceived benefits of integrating AR. Inspired from the Task-technology Fit model introduced by [3] which aims to assess the match between the task and technology characteristics, this research proposes a comprehensive map that outlines the relationships between the capabilities of $A R$, the identified AR use-cases, and the potential perceived benefits associated with AR. While users can experience AR through Head-Mounted Displays (HMD) or Hand-Held Displays such as mobiles and tablets, HMD provides the user with more flexibility as they enable hand-free operations [4]. Additionally, a study conducted by [5] surveyed 128 construction professionals and showed that HMD are becoming more commonly used in the construction industry. Thus, the comprehensive map presented in this paper is developed with HMD in mind.

\section{Components of the comprehensive map}

The comprehensive map has three main components: AR Use-Cases, AR capabilities and AR Potential Benefits. The following sections will identify and explain each of these three components.

\subsection{Augmented reality use-cases in the construction phases}

In recognition of the potential of AR in construction, researchers have conducted several reviews of ARrelated efforts in the industry. Numerous efforts have been undertaken to explore AR use-cases and investigate ways to integrate the technology into construction. Some studies have focused on a certain phase of the construction lifecycle such as design, pre-construction, construction, and operation and maintenance [6-9]. Other research endeavours have conducted a comprehensive assessment of AR usecases throughout the project life-cycle - from conceptual planning to decommissioning. This paper builds on the work of $[5,10,11]$ and presents 23 AR-use cases for the construction phase of a project. The 23 AR use-cases are outlined alphabetically in the middle column of Fig. 5.

\subsection{Augmented reality capabilities}

Prior to integrating AR into a use-case (i.e. task), it is important to analyze the extent to which AR can support this use-case. Davenport (1993) explained that opportunities for supporting a process with Information technology (IT) fall into nine categories. The opportunities to integrate AR - an emerging and promising technology in the realm of IT - into a use-case can be also lumped into those nine categories, defining the capabilities of AR, as explained (in an alphabetical order) below:

C1 Analytical: The analytical capability is related to decision making enhancement and improving information analysis. In addition to providing real time in-situ information to visualize data [13] AR provides a platform to visualize and interact with data leading to better user cognition and environment perceptive[14].

C2 Automation: The automation capability is described as reducing human labour through automating different tasks. AR systems have the ability to automate processes by generating information automatically in real-time and visualizing it in a real construction working environment [15].

C3 Disintermediating: The disintermediating capability is best described as removing intermediaries from activities. AR is one of the digital era technologies with an ability to add or remove intermediator processes [16]. For example, using AR can substitute the processes of manually capturing, storing and analyzing data.

C4 Geographical: The geographical capability is related to coordinating activities and taking decisions across distances, irrespective of the location of decision makers. AR promotes new types of collaborative interfaces to enhance face-to-face and remote collaboration [17]. 
C5 Informational: The informational capability assists in understanding a process. AR offers opportunities to display, capture and store information for later analysis [18].

C6 Integrative: The integrative capability is the coordination between tasks and processes. AR can capture and generate context-rich data that facilitated the coordination between cross-functional teams [19].

C7 Intellectual: The intellectual capability is the capturing and distribution of intellectual assets. AR supports tacit knowledge exchange, allowing remote expert, for example, to transfer their knowledge through the AR medium using demonstrations such as graphics, audios, and videos [20].

C8 Sequential: The sequential capability is related to changing the sequence of processes and/or enabling parallelization. AR systems, enabled with remote collaboration, allow different activities/tasks to be performed in parallel [15].

C9 Tracking: The tracking capability is related to the close monitoring of process status and objects. AR superimposes digital models onto the real world, allowing users to track progress [21].

\subsection{Augmented Reality Potential Benefits}

The wide range of AR use-cases and the evolution of the capabilities of the technology highlight a new era for the construction industry [9]. The suitability of a technology and the success of its integration are manifested by its benefits. This study adopted the benefits identified in [5] but excluded two of them: "Improving the corporate image" and "Improving growth and success by creating new business models". The rational for removing these two benefits stems from the fact that this paper focuses on the construction phase and these two benefits are long-term benefits that impact the organization rather than the construction site. The 14 AR potential benefits are outlined in the rightmost column of Fig. 5.

\section{Examples illustrating the concept of comprehensive map}

This section includes some applications of AR on construction sites to illustrate the concept of the comprehensive map. After an explanation of the applications, the connected capabilities and benefits are explained.

\subsection{AR Application 1: Remote Expert System (RES)}

A remote expert system (RES) connects a technician on the construction site with one or more remote experts sitting in their offices. In a simple version, the person on the construction site has glasses with a small screen and a small video camera - like Doka's remote instructor [22]. The remote expert has access to a live-video stream from the construction site and can give written or voice advices that are transferred onto the small screen. Two of the authors have previously worked on a more advanced RES that equips the technician with AR glasses or a tablet and the remove expert with a computer program. Fig. 1 shows this remote expert system in the left picture. The remote expert program is displayed on the monitor and is featured with a live-video stream and the option to create markings. The upper left corner reflects the view of the HMD device of the technician. The application of RES is extended with the use of a camera to the generation of a 3D environment model (see Fig. 1, right image). The remote expert can switch from the live view to a $3^{\text {rd }}$ person view, in which the 3D environmental model is shown

Multiple AR use-cases are supported through this AR application, particularly, UC14 (real-time support of field personnel) and UC16 (remote site inspection). Fig. 2 shows the corresponding AR capabilities and potential benefits for AR use-case UC14.
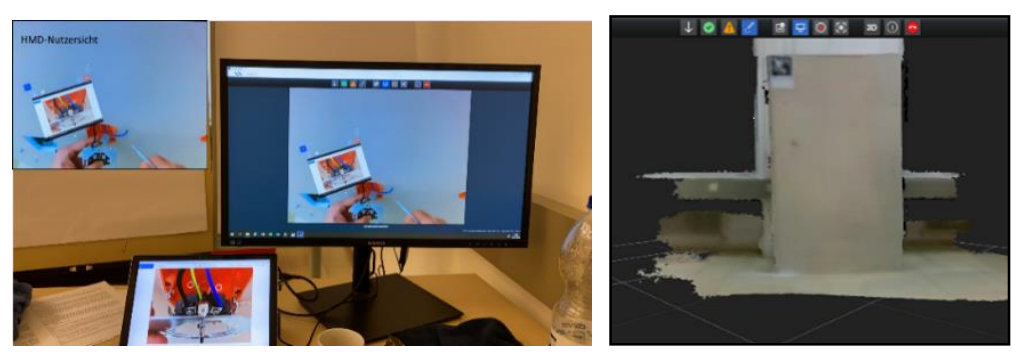

Fig. 1. Remote site inspection with Remote Expert System 


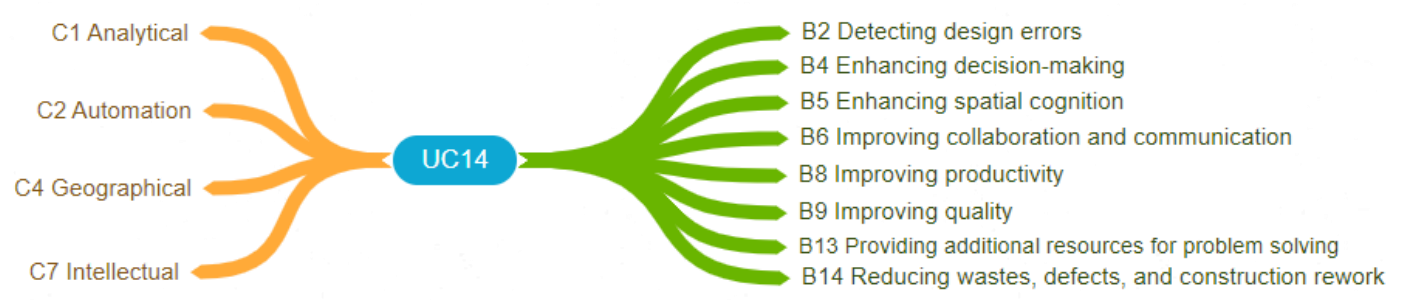

Fig. 2. AR capabilities and benefits connected with UC14 (generated using Coggle.it)

\subsection{AR Application 2: Doka Verification Buddy with Augmented Reality and Artificial Intelligence}

The formwork company Doka is developing a formwork verification buddy for its system formwork Frami Xlife [22]. This AR system shows the right locations of the clamps, the fixing bolts and the corner connectors for the formwork (Fig. 3). Additionally, an Artificial Intelligent system checks if any of these connection parts are missing - a challenging task given that the parts have the same colour as the frame of the formwork. The system marks the correctly placed parts in green and the missing parts in red, supporting unexperienced workers when erecting the formwork. The Doka Verification Buddy can, therefore, be used for detecting errors as well as for training purposes. The system is still in its development stage and is expected to be developed for HMD.

The Doka verification buddy supports UC8 (on-site inspection). The corresponding capabilities and benefits for this use case are shown in Fig. 4. It should be noted that while benefits B6 and B10 apply to UC8, they do not apply to the current version of the Doka verification buddy.

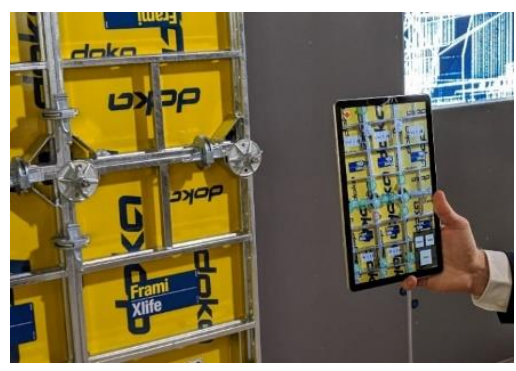

Fig. 3. Doka Verification Buddy: Visualization of augmented work instructions in the field in combination with Al

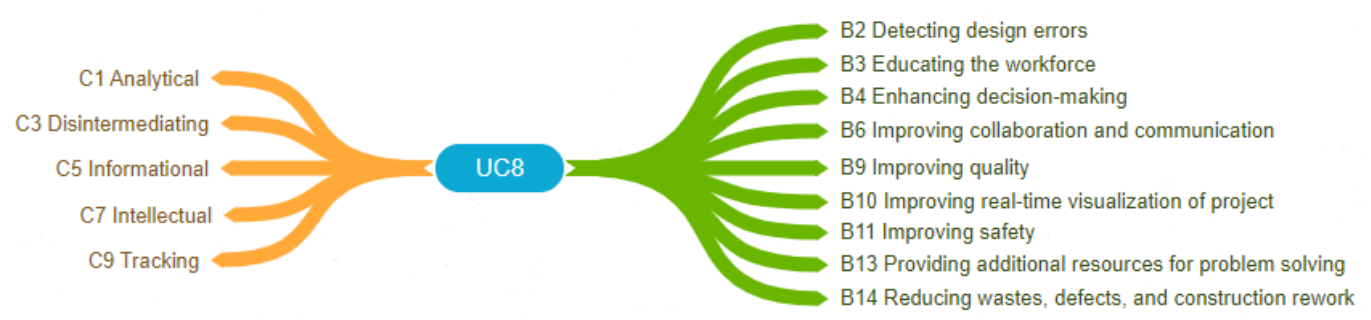

Fig. 4. AR capabilities and benefits connected with UC8 (generated using Coggle.it)

\section{Comprehensive map}

The culminating effort of this paper is a comprehensive map (Fig. 5) that outlines the relationships between 23 AR use-cases in the construction phase, the nine different capabilities of AR, and the 14 AR potential benefits. Fig. 5 shows that while the nine AR capabilities are essential to the integration of the technology into construction use-cases, Automation (C2), Geographical (C4), and Information (C5) are three capabilities that are applicable to most of the 23 AR use-cases. Additionally, Enhancing decision-making (B4), Improving Collaboration and Communication (B6), Improving Productivity (B8), Providing additional resources for problem solving (B13), and Reducing wastes, defects, and construction rework (B14) are found to be the most frequently expected benefits. 


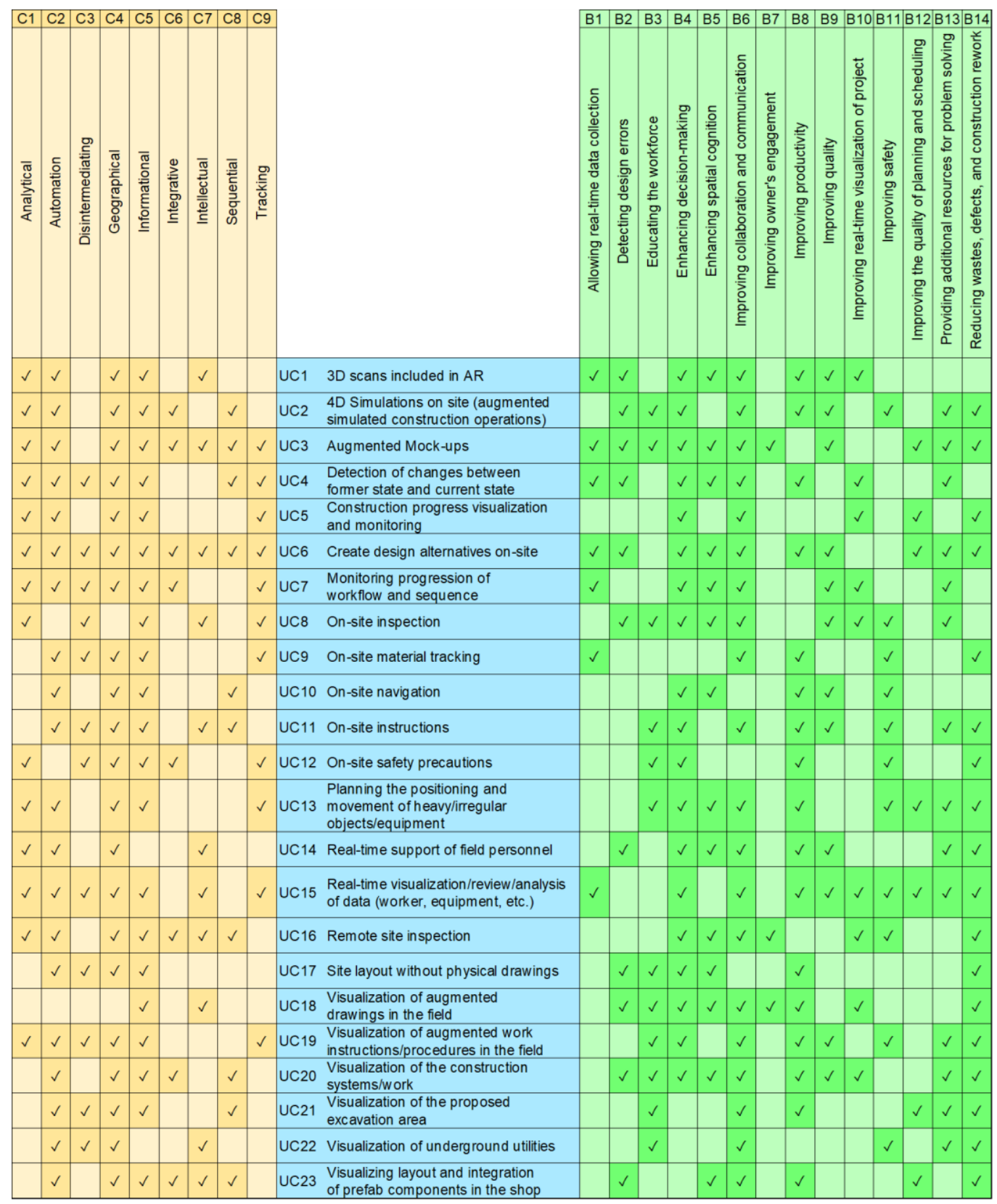

Fig. 5: Comprehensive Map

\section{Conclusions and further studies}

As interest in AR continues to grow in the construction industry, it is important to explore the suitability of the technology. This study focuses on the opportunities and benefits of integrating AR into the construction phase of a project. Twenty-three AR use-cases were extracted from the literature, nine AR capabilities were discussed, and 14 AR potential benefits were identified. Based on the Task-Technology Fit theory, this study developed a comprehensive map that outlines the relationships between each of the 23 AR use-cases and their corresponding AR capabilities and perceived benefits. The map revealed that Automation, Geographical, and Information are the most commonly used AR capabilities during the construction phase. The results also showed that Enhancing decision-making, Improving Collaboration and Communication, Improving Productivity, Providing additional resources for problem solving, and Reducing wastes, defects, and construction rework are the five AR benefits that are frequently perceived to result from the integration of AR into the construction use-cases. This paper contributes to the AR implementation roadmap by providing industry practitioners an understanding of the capabilities and benefits for integrating AR into construction tasks. Further research could expand the scope of work and examine AR use-cases throughout the lifecycle of a project and their relationships to the AR capabilities and AR potential benefits. Researchers 
could also build on the comprehensive map presented in this study and develop prototypes to validate the outlined relationships.

\section{References}

[1] PricewaterhouseCoopers, Global Construction 2030. https://www.pwc.com.tr/en/hizmetlerimiz/altyapi-yatirimlari/yayinlar/pwc-globalconstruction-2030.html

[2] Nassereddine, H., Veeramani, D. and Hanna, A., 2019. Augmented Reality-Enabled Production Strategy Process. In: Proceedings of the 36th International Symposium on Automation and Robotics in Construction (ISARC). Available at: https://doi.org/10.22260/isarc2019/0040.

[3] Goodhue, D.L., 1995. Understanding User Evaluations of Information Systems. Management Science, 41(12), pp.1827-1844. Available at: https://doi.org/10.1287/mnsc.41.12.1827.

[4] Chalhoub, J. and Ayer, S.K., 2017. Mixed Reality for Electrical Prefabrication Tasks. Computing in Civil Engineering 2017. Available at: https://doi.org/10.1061/9780784480847.010.

[5] Nassereddine, H.M., 2019. Design, Development and Validation of an Augmented Reality-Enabled Production Strategy Process for the Construction Industry. Ph.D. at the University of Wisconsin-Madison.

[6] Shin, D.H. and Dunston, P.S., 2008. Identification of application areas for Augmented Reality in industrial construction based on technology suitability. Automation in Construction, 17(7), pp.882-894. Available at: https://doi.org/10.1016/j.autcon.2008.02.012.

[7] Ghaffarianhoseini, A., Doan, D., Zhang, T., Rehman, A.U., Naismith, N., Tookey, J., Rotimi, J., Ghaffarianhoseini, A., Yu, T., Mu, Z. and Qi, Y., 2016. Integrating Augmented Reality and Building Information modelling to Facilitate Construction Site Coordination. In: Proceedings of the 16th International Conference on Construction Applications of Virtual Reality.

[8] van Berlo, L., Helmholt, K.A. and Hoekstra, W., 2009. C2B: Augmented Reality on the Construction Site. In: Proceedings of the 9th International Conference on Construction Applications of Virtual Reality.

[9] Kivrak, S. and Arslan, G., 2018. Using Augmented Reality to Facilitate Construction Site Activities. Advances in Informatics and Computing in Civil and Construction Engineering, pp.215-221. Available at: https://doi.org/10.1007/978-3-030-00220-6_26.

[10] Goger, G., Piskernik, M. and Urban, H., 2018. Studie: Potenziale der Digitalisierung im Bauwesen. WKO.

[11] Urban, H., Schranz, Ch. and Gerger, A., 2019. BIM auf Baustellen mit Augmented Reality. Bauaktuell 10, pp. $192-196$.

[12] Davenport, T.H., 1993. Process innovation: reengineering work through information technology. Harvard Business Press.

[13] ElSayed, N.A., Thomas, B.H., Smith R.T., Marriott, K. and Piantadosi, J., 2015. Using augmented reality to support situated analytics. 2015 IEEE Virtual Reality (VR). Available at: https://doi.org/10.1109/vr.2015.7223352.

[14] Luboschik, M., Berger, P. and Staadt, O., 2016. On spatial perception issues in augmented reality based immersive analytics. In: Proceedings of the 2016 ACM Companion on Interactive Surfaces and Spaces, pp. 47-53. Avaialble at: https://doi.org/10.1145/3009939.3009947

[15] Verlinden, J., Horváth, I. and Nam, T.J., 2009. Recording augmented reality experiences to capture design reviews. International Journal on Interactive Design and Manufacturing (IJIDeM), 3(3), pp.189-200. Available at: https://doi.org/10.1007/s12008-009-00748.

[16] Miller, R. and Custis, K.R,. 2017. Disruption. Digitalization. Disintermediation. EY.

[17] Billinghurst, M. and Kato, H., 2002. Collaborative Augmented Reality. Communications of the ACM, 45(7), pp.64-70. Available at: https://doi.org/10.1145/514236.514265.

[18] Diaz, C., Hincapié, M. and Moreno, G., 2015. How the Type of Content in Educative Augmented Reality Application Affects the Learning Experience. In: Proceedings of Computer Science, 75, pp.205-212. Available at: https://doi.org/10.1016/j.procs.2015.12.239.

[19] Biron, J. and Lang, J., 2018. Unlocking the Value of Augmented Reality Data. MIT SMR. Avaialble at: https://sloanreview.mit.edu/article/unlocking-the-value-of-augmented-reality-data/.

[20] Aromaa, S., Väätänen, A., Aaltonen, I. and Heimonen, T., 2015. A model for gathering and sharing knowledge in maintenance work. In: Proceedings of the European Conference on Cognitive Ergonomics 2015 - ECCE '15. Available at: https://doi.org/10.1145/2788412.2788442.

[21] Wang, X. and Love, P.E., 2012. BIM + AR: Onsite information sharing and communication via advanced visualization. In: Proceedings of the 2012 IEEE 16th International Conference on Computer Supported Cooperative Work in Design (CSCWD). Available at: https://doi.org/10.1109/cscwd.2012.6221920.

[22] Doka 2019. Doka Campus: Die Welt der Schalung mit allen ihren Facetten auf der bauma 2019. Avaialble at: https://www.doka.com/at/news/press/Doka_Campus_2019. 